\title{
Retained faecalith following laparoscopic appendectomy
}

\author{
Yegi Kim ${ }^{1}$, Joseph Kong², Evan Williams², and Satish Warrier² \\ ${ }^{1}$ Peninsula Health \\ ${ }^{2}$ Alfred Health
}

August 28, 2020

\begin{abstract}
There have been a few case studies showing intra-abdominal abscesses due to retained faecalith post laparoscopic appendectomy. A 29 years-old woman presented with right lateral abdominal wall and pelvic collection due to retained faecalith post interval laparoscopic appendectomy for perforated appendicitis. She underwent exploration for faecalith retrieval successful outcome.
\end{abstract}

\section{Introduction}

Laparoscopic appendectomy is currently the first choice of surgical approach for an acute appendicitis due to lower rate of surgical site infections, shorter hospital stay and better cosmetic outcome $(1,2,3)$. Furthermore, an interval appendicectomy is favoured by many surgeons for treatment of perforated appendicitis. However, one of the complications from laparoscopic appendectomy includes intra-abdominal abscess formation secondary to retained faecalith $(1,4)$. Although rare, a dropped faecalith can occur during appendectomy or due to expulsion from perforated appendix. We present a case of recurrent intra-abdominal abscesses from a retained faecalith in the intra-muscular layers of the iliacus in a patient whose initial diagnosis of an acute appendicitis was delayed.

\section{Case Report}

A 29 years-old woman initially presented with a 2 week-history of non-migratory right iliac fossa pain with subjective fever. The patient's history of complaint was not typical of acute appendicitis. After unremarkable inflammatory markers and pelvic ultrasound that did not visualise the appendix, she was discharged home with a provisional diagnosis of pelvic inflammatory disease and was given oral antibiotics for seven days.

However, she represented to our emergency department (ED) with worsening abdominal pain, ongoing fever and mild nausea. A computed tomography (CT) was organised and it showed a perforated appendicitis with retrocaecal abscess and a calcified appendicolith. She then had an ultrasound-guided drainage of the abscess and was discharged home with a view of performing an interval laparoscopic appendectomy. After discharge, the patient returned to ED for persistent recurrence of a right pelvic collection involving the right iliacus and lateral abdominal wall muscle. This was again radiologically drained. A colonoscopy was performed during this presentation to exclude any primary tumours before surgery. The entire colon was examined and showed normal mucosa and appendiceal orifice.

The patient then underwent a semi-emergent laparoscopic appendectomy. During the procedure, thorough abdominal wash was performed and a drain tube placed in the right iliac fossa. The patient had an uneventful post-operative recovery, with normalisation of inflammatory markers and the drain tube removed. She was then discharged home with oral antibiotics. During her follow-up in the general surgery clinic, the histopathology reported chronic appendicitis with granulomas but the faecalith was not within the specimen. On review, it was noted that she had intermittent abdominal discomfort and a repeat of C-reactive protein 
(CRP) showed that it has elevated to 41. A repeat CT scan was performed which showed re-accumulation of the right pelvic and lateral abdominal collection with the retained faecalith (figures 1-2).

Although the abscess was intramuscular, the faecalith and the pelvic abscess were going through the abdominal wall into the subcutaneous fat (figure 3). There was a brief discussion with an interventional radiologist for a hook-wire insertion preoperatively for direct localisation of the faecalith but given the location of the abscesses, intraoperative ultrasound was used instead. The patient underwent exploration and surgical removal of retained faecalith through a right lateral hip approach (figure 4). A drain was inserted into the cavity and the wounds were closed with interrupted sutures.

\section{Outcome/follow up}

The wound was complicated by formation of seroma, which was managed conservatively. The patient was reviewed again six weeks post-operation and remained well with no further complications of her wound or pelvic abscess drainage.

\section{Discussion}

An interval appendectomy for perforated appendicitis with established abscesses is widely practiced by many surgeons (5). It has been suggested that an interval appendectomy has many benefits including reducing the overall rate of adverse events (6). The concept of delaying surgery would allow the intra-peritoneal contamination and inflammation to settle down with antibiotics, allowing surgeons to mitigate the risk of an unfriendly plane due to ongoing sepsis (6). However, the strategy of an interval appendectomy was not successful in our case due to an infective nidus, a retained faecalith. This particular complication, although not common, is a well-known complication after a laparoscopic appendectomy. The risk of retained faecalith is even higher in a case of perforated appendicitis (7). The faecalith may drop from the base of the appendix when it is being resected or when the appendix is being extracted through the port $(7,8)$. A study suggests different strategies to prevent faecalith spillage including gentle manipulation of an appendix and use of an endoscopic bag to retrieve the appendix (9). We suspect that in our case the retained faecalith will likely have migrated from the perforated appendix into the intramuscular layers of iliacus post percutaneous drainage of the abscess.

There have been different recommendations for the management of a retained faecalith $(1,10)$. Black et al. presented a case of an abscess with faecalith adequately managed with intravenous antibiotics only (4). Some studies suggest percutaneous drainage and extraction of faecalith $(4,10)$. Despite these, surgical removal of faecalith is recommended by many surgeons (1). We had two rationales for a right lateral hip approach instead of intra-abdominal approach. Firstly the location of the abscess was continuous from subcutaneous plane to intraabdominal plane. Secondly the planes of dissection and retrieving the faecalith would be difficult if through intra-abdominal. Hence, taking a new approach through virgin territory has aid with the recovery of the faecalith. Although she developed seroma post operatively, she recovered relatively quickly after the operation.

This case highlights the importance of identifying and extracting a retained faecalith to decrease the morbidity associated with a persistent infective nidus. Given that the patient had a laparoscopic procedure with a failed attempt, we have decided on a different surgical access, right lateral hip approach, with the intention of a midline laparotomy if not successful. This has led to a successful resolution of her right pelvic abscess formation with no added surgical morbidity.

\section{Acknowledgements}

Nil financial support

\section{Ethics}

Patient's informed and signed consent obtained

\section{References}


1. Gamble L, Saze A. 2016. Chronically retained fecalith following laparoscopic appendectomy. Surgical infections case reports 1(1): 35-37.

2. Hori T, Machimoto T, Kadokawa Y, Hata T, Ito T, Kato S, Yasukawa D, Aisu Y, Kimura Y, Sasaki M, Takamatsu, Kitano T, Hisamori S, Yoshimura T. 2017. Laparoscopic appendectomy for acute appendicitis: How to discourage surgeons using inadequate therapy. World Journal of Gastroenterology 23(32): 5849-5859.

3. Kim N, Reed W, Abbas M, Katz D. 2004. CT Identification of Abscesses After Dropped Appendicoliths During Laparoscopic Appendectomy. American Journal of Roentgenology 182:1203-1205.

4. Black M, Ha BY, Kang YS, Garland A. 2013. Perihepatic abscess caused by dropped appendicoliths following laparoscopic appendectomy: Sonographic finding. Journal of Clincal Ultrasound 1:366-369.

5. Weiner D, Katz A, Hirschl R, Drongowski R, Coran A. 1995. Interval appendectomy in perforated appendicitis. Paediatric Surgery International 10:82-85.

6. Kim IY. 2016. Minimally Invasive Interval Appendectomy for Perforated Appendicitis With a Periappendiceal Abscess. Annals of Coloproctology 32(3): 88-89.

7. Katagiri H, Ishitani M, Sakamoto T, Yoshinaga Y, Kubota T, Miyabe A. 2013. Retained fecaliths after laparoscopic appendectomy disappearing spontaneous with non-operative management. Edorium Journals 4(11):650-653.

8. Rasuli P, Friendlich MS, Mahoney JE. 2007. Percutaneous Retrieval of a Retained Appendicolith. Cardiovascular Interventional Radiology 30(2):342-4.

9. Strathern DW, Jones BT. 1999. Retained fecalith after laparoscopic appendectomy. Surg Endosc 13(3):287-9.

10. Singh AK, Hahn PF, Gervais D, Vijayraghavan G, Mueller PR. 2008. Dropped appendicolith: CT findings and implications for management. American Journal of Roentgenology 190(3):707-11.

\section{Figures}

Figure 1.

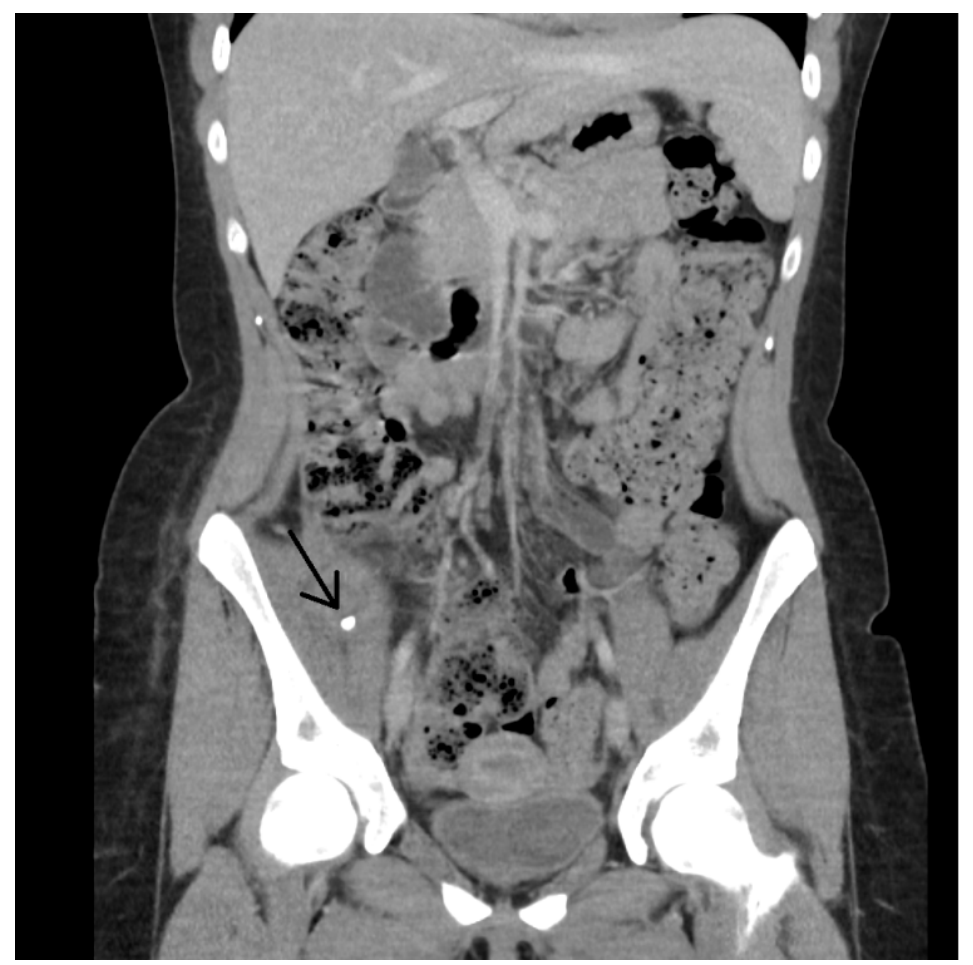


CT abdomen showing the retained faecalith.

Figure2.

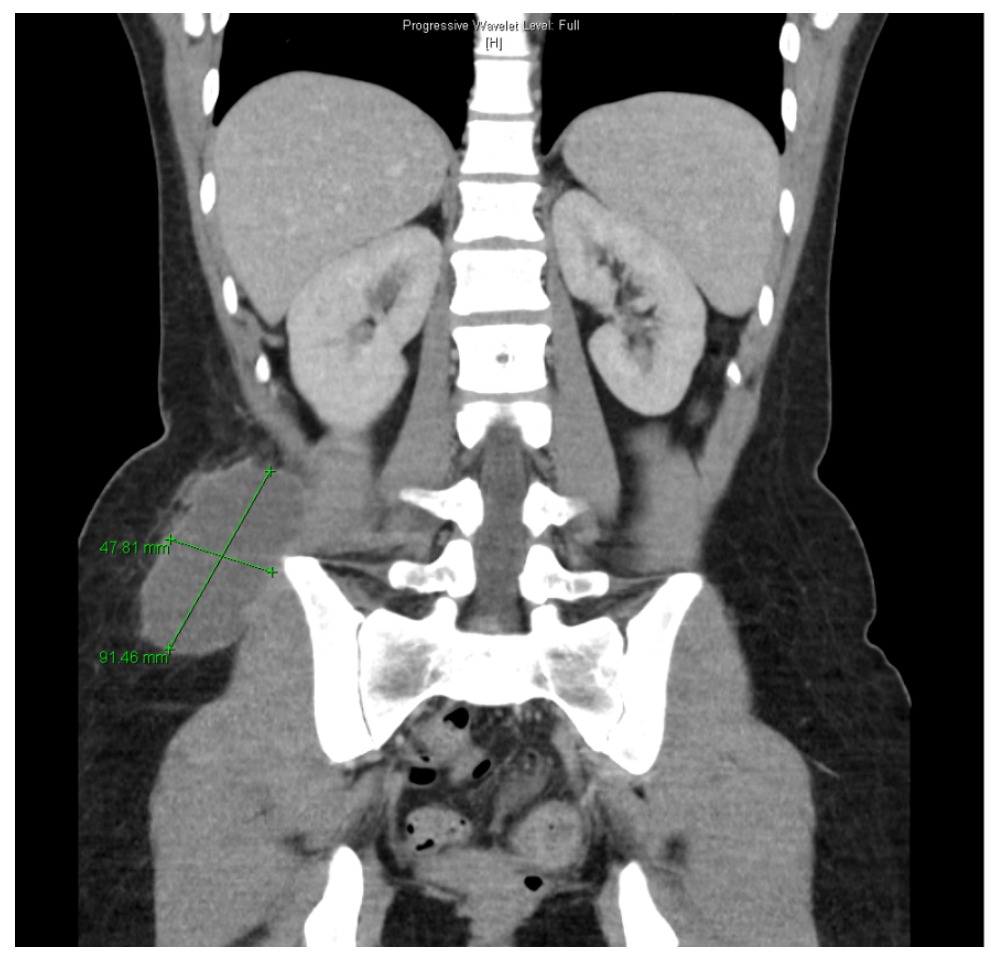

CT abdomen showing right lateral abdominal wall collection.

Figure 3. 


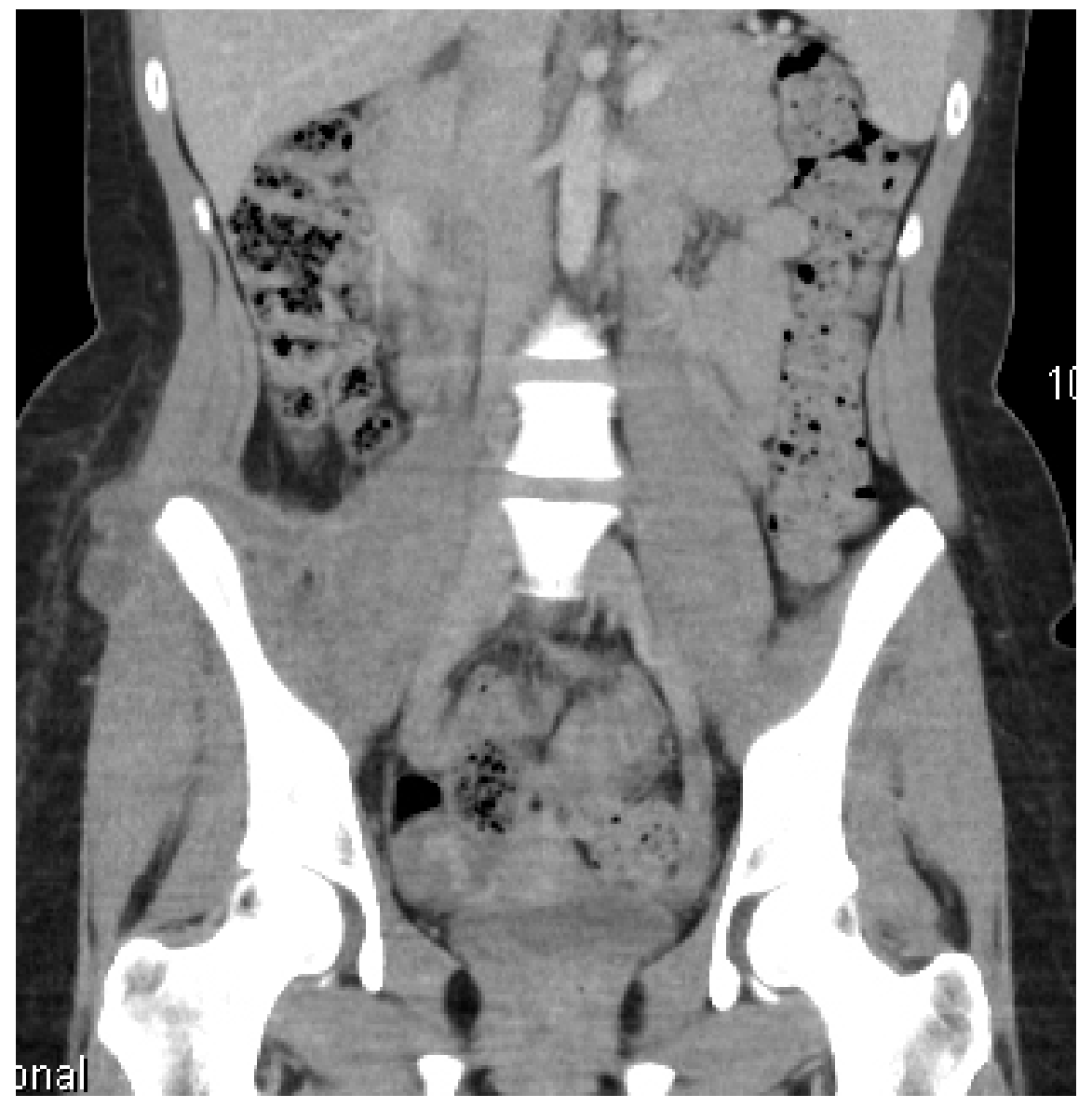

CT abdomen showing horseshoe shaped collection in right lateral abdominal wall into the subcutaneous fat. Figure 4. 


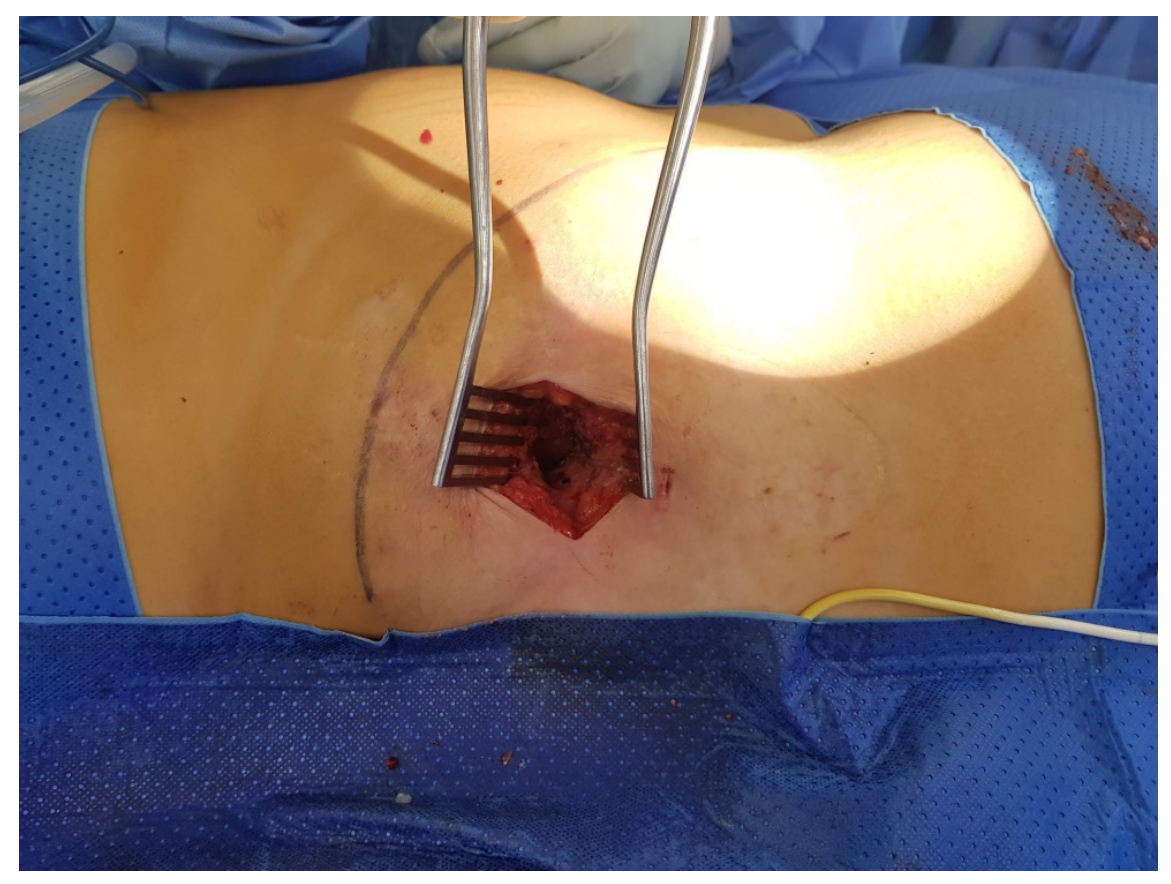

Intra-operative view of exploration. 


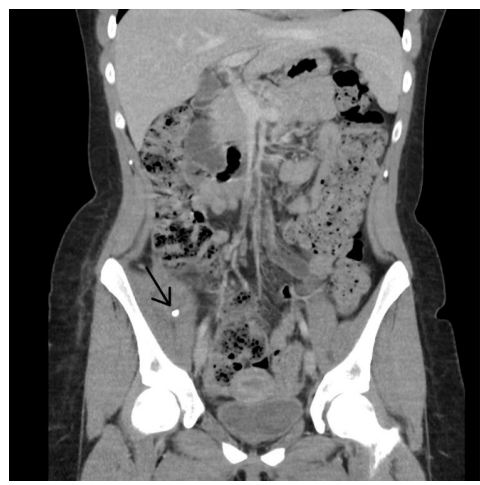

$\mathrm{CT}$ abdomen showing the retained faecalith 


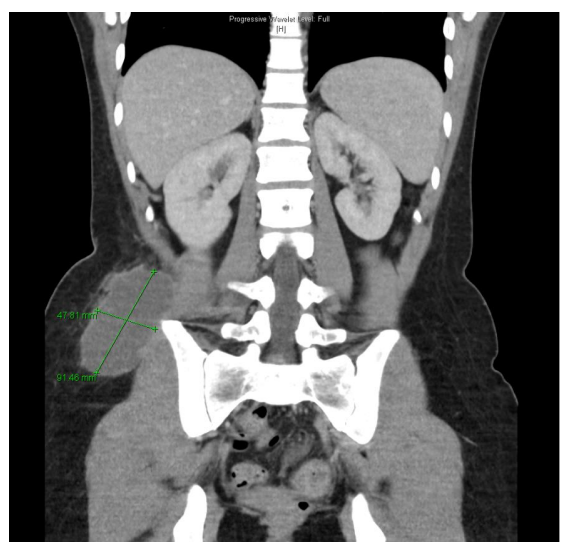

$\mathrm{CT}$ abdomen showing right lateral abdominal wall collection 


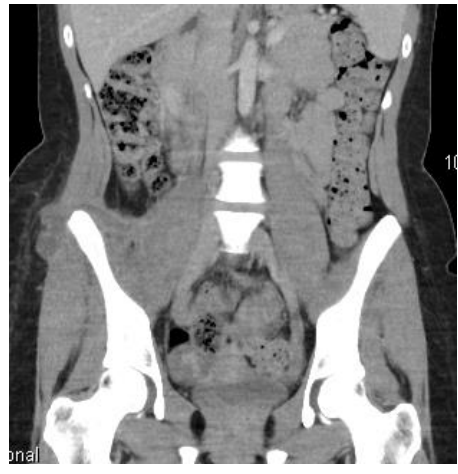

CT abdomen showing horseshoe shaped collection in right lateral abdominal wall into the subcutaneous fat 


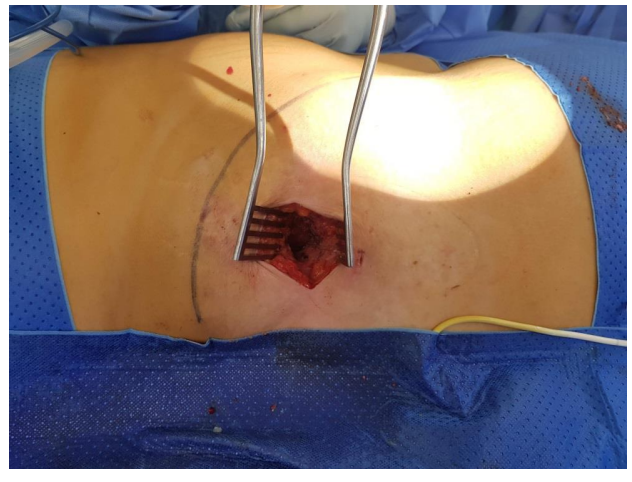

Intra-operative view of exploration 